\title{
Análise do conceito de conforto: contribuições para o diagnóstico de Disposição para Conforto melhorado
}

\author{
Analysis of the concept of comfort: contributions to the diagnosis of Readiness for enhanced comfort \\ Análisis del concepto de confort: contribuciones al diagnóstico de la Disposición para mejorar el confort
}

\author{
Cristiane Soares Carius Nogueira Pereira ${ }^{1}[1$ \\ Claudia Angélica Mainenti Ferreira Mercês ${ }^{1}$ (B) \\ Rafael Oliveira Pitta Lopes $^{1}$ (D) \\ Jackeline Felix de Souza ${ }^{1}$ (1) \\ Jaqueline da Silva Soares Souto ${ }^{1}$ (]) \\ Marcos Antônio Gomes Brandão ${ }^{1}$ (C)
}

1. Universidade Federal do Rio de Janeiro, Programa de Pós-graduação em Enfermagem. Rio de Janeiro, RJ, Brasil.
Autor correspondente:

Cristiane Soares Carius Nogueira Pereira. E-mail: cristianekarius@hotmail.com

Recebido em 16/07/2019.

Aprovado em 01/11/2019.

\section{RESUMO}

Objetivos: Analisar o conceito de conforto à luz do modelo de análise conceitual evolucionista de Rodgers e incorporar elementos da análise conceitual na estrutura do diagnóstico de enfermagem "Disposição para conforto melhorado" (00183) Métodos: Estudo de análise de conceito com aplicação das sete etapas do modelo evolucionário de Rodgers, sendo orientado para a condução da fase 1 de validação do diagnóstico de enfermagem. O material para análise foi obtido com a estratégia de revisão integrativa da literatura. Resultados e discussão: Apresenta-se perspectiva histórico-desenvolvimental do conceito de quatro períodos temporais. Evidencia atributos, antecedentes, consequentes, termos substitutos e definições conceituais obtidos da decomposição conceitual, utilizando-os para a atualização dos componentes da "Disposição para conforto melhorado" (00183). Conclusão e implicações para a prática: A abordagem evolucionária de análise do conceito mostrou ser apropriada para clarificar o conceito de conforto, dela emergindo tanto uma perspectiva orientada para o bem-estar quanto para as alterações do desconforto. A análise do conceito de conforto no método evolucionário contribuiu para a atualização do diagnóstico de enfermagem em tela. Os resultados obtidos fornecem elementos para a realização de futuros estudos de validação diagnóstica por experts e de validação clínica o que impacta positivamente no uso da linguagem diagnóstica.

Palavras-chave: Formação de Conceito; Conforto do Paciente, Diagnóstico de Enfermagem.

\section{ABSTRACT}

Objectives: To analyze the concept of comfort in light of Rodgers' conceptual evolutionary analysis model and to incorporate elements of conceptual analysis into the framework of the nursing diagnosis "Readiness for enhanced comfort" (00183). Methods: Concept analysis with the application of the seven steps of Rodgers' evolutionary model, oriented to the conduction of phase 1 of the validation of the nursing diagnosis. The material for analysis was obtained through the strategy of integrative review of the literature. Results and discussion: A historical-developmental perspective of the concept with four tempora periods is presented. It reveals attributes, antecedents, consequences, substitute terms and conceptual definitions obtained from conceptual decomposition and we used them to update the components of the diagnosis "Readiness for enhanced comfort" (00183). Conclusion and implications for practice: The evolutionary approach to concept analysis proved to be appropriate to clarify the concept of comfort, and a well-being perspective and a discomfort perspective emerged from it. An analysis of the concept of comfort in accordance with the evolutionary method contributed to the update of the nursing diagnosis. The results provide elements for future diagnostic validation studies conducted by experts and clinical validation studies, which has a positive impact on the use of diagnostic language.

Keywords: Concept Formation; Patient Comfort; Nursing Diagnosis.

\section{RESUMEN}

Objetivos: Analizar el concepto de confort a la luz del modelo de análisis conceptual evolutivo de Rodgers e incorporar elementos de análisis conceptual en el marco del diagnóstico enfermero "Disposición para mejorar el confort” (00183). Métodos: Estudio de análisis conceptual con aplicación de los siete pasos del modelo evolutivo de Rodgers, orientado a realizar la fase 1 de validación del diagnóstico enfermero. El material para el análisis se obtuvo con la estrategia de revisión integradora de la literatura. Resultados y discusión: Se presenta una perspectiva histórica y de desarrollo del concepto de cuatro períodos temporales. Destaca los atributos, antecedentes, consecuencias, términos sustitutos y definiciones conceptuales obtenidas de la descomposición conceptual, usándolos para actualizar los componentes de la "Disposición para mejorar el confort" (00183). Conclusión e implicaciones para la práctica: se ha demostrado que el enfoque evolutivo del análisis de conceptos es apropiado para aclarar el concepto de confort desde una perspectiva orientada hacia el bienestar hasta los cambios de la incomodidad. El análisis del concepto de confort en el método evolutivo contribuyó a la actualización del diagnóstico enfermero. Estos resultados proporcionan elementos para futuros estudios de validación diagnóstica por expertos y validación clínica, lo que impacta positivamente el uso del lenguaje diagnóstico.

Palabras clave: Formación de Concepto; Comodidad del Paciente; Diagnóstico de Enfermería 


\section{INTRODUÇÃO}

O conforto é um conceito que tem sido identificado como elemento historicamente associado aos cuidados de enfermagem assumindo diferentes significados que se conectam a mudanças políticas, tecno-científicas, sociais e religiosas da humanidade, guardando relação com as ciências da saúde e a enfermagem em particular. ${ }^{1,2}$

Embora observações empíricas da prática da enfermagem e os achados da literatura apontem o conforto como integrante dos cuidados de enfermagem, ainda são muitas as indagações sobre sua conformação como fenômeno e quais os conceitos a ele relacionados. A relevância do conceito e da teorização na enfermagem incentivou mais estudos, especialmente a partir das contribuições de Janice Morse e Katherine Kolcaba. ${ }^{3-7}$

Entretanto, o interesse pelo conforto não ocorre apenas no nível teórico-conceitual, atraindo a atenção dos pesquisadores e clínicos para os sistemas de classificação de fenômenos diagnósticos de enfermagem. Na Classificação Internacional da Prática de Enfermagem (CIPE) existem três termos relacionados ao conforto classificados no eixo foco (Conforto, Desconforto e Sinal de desconforto) e um termo classificado como diagnóstico/resultado (Desconforto). ${ }^{8} \mathrm{Na}$ classificação diagnóstica da NANDA International, Inc. (NANDA-I) existem dois diagnósticos: "Conforto prejudicado" (00214) e "Disposição para conforto melhorado" (00183) incorporados no Domínio 12: conforto e nas classes 1, 2, 3: conforto físico, ambiental e social. ${ }^{9}$

Nas pesquisas diagnósticas de enfermagem a investigação tem se concentrado nas situações de alteração do conforto e desconforto, não sendo encontrados artigos em revistas indexadas que abordem o diagnóstico de enfermagem (DE) de promoção da saúde "Disposição para conforto melhorado" (00183). Inclusive as referências indicadas pela NANDA Internacional, Inc. para a "Disposição para conforto melhorado" (00183) não apresentam estudos de validação diagnóstica, estando concentrados nas análises conceituais e estudos teóricos do conforto, o que pode indicar a necessidade de melhor refinamento conceitual antes do início dos estudos clínicos. ${ }^{9}$

O diagnóstico "Disposição para conforto melhorado" (00183) refere-se a um diagnóstico de promoção da saúde introduzido na Taxonomia II da NANDA-I em 2006 e revisado em 2013, situa-se em nível de evidência (LOE) de 2.1, ou seja, contém título, definição, e características definidoras, tendo uma literatura citada para a definição, bem como para cada uma das características definidoras. ${ }^{9}$ Sua elevação ao nível de evidência 2.2 exige que a primeira etapa dos estudos de validação, a análise de conceito, seja realizada de modo a "demonstrar a existência de um conjunto de conhecimentos substancial em apoio ao diagnóstico". 9

Os autores deste estudo reconhecem os avanços já obtidos com as análises conceituais acerca do conforto e destacam estudo de análise conceitual publicado em 2002 que utilizou o método evolucionário de Rodgers para desenvolvimento do conceito. ${ }^{10}$ Entretanto, a necessidade de desenvolver continuamente o conceito de conforto e os diagnósticos de enfermagem é um aspecto central para o avanço do corpo de conhecimento disciplinar. A inclusão do diagnóstico de enfermagem de "Disposição para conforto melhorado" (00183) após o ano de publicação do mencionado estudo motivou uma nova investigação adotando-se o método evolucionário. Também, parte-se da consideração de que os processos de validação de diagnósticos de enfermagem exigem análises de conceitos antes da validação por experts e validação clínica. ${ }^{11}$

Diante disso, são objetivos do estudo: analisar o conceito de conforto à luz do modelo de análise conceitual evolucionista de Rodgers e incorporar elementos da análise conceitual na estrutura do diagnóstico de enfermagem "Disposição para conforto melhorado" (00183).

\section{REVISÃO DA LITERATURA}

A origem do desenvolvimento dos conceitos na literatura da enfermagem coincide com o maior desenvolvimento das teorias de Enfermagem. ${ }^{12}$ Os conceitos são a base da ciência, sendo considerados os menores elementos com os quais se constroem as teorias científicas, definindo os fenômenos a serem investigados, bem como diferindo uma ciência de outra, por isso, cada ciência tem seu conjunto próprio de conceitos, que determinam os campos de ação, os métodos, os temas, os objetivos e seus objetos de pesquisa. ${ }^{13}$

Os conceitos também podem ser considerados como representações cognitivas abstratas da realidade perceptível formada por experiências diretas ou indiretas. O método de análise de conceito é usado para definir os conceitos existentes e tem sua raiz nos escritos de Aristóteles (Século IV a.C.) sendo o primeiro a estabelecer o processo de definição mediante uma atividade científica fundamentada. Assim, legitimou esforços para analisar e definir conceitos com o objetivo de demonstrar que eles são abstrações constituídos de características essenciais e inalteradas de elementos ou objetos no mundo. ${ }^{14}$

O alicerce teórico da ciência da enfermagem se estrutura em um processo dinâmico, que tem como movimento o surgimento na prática e que se reproduz na pesquisa, particularmente a partir da análise de conceitos e do desenvolvimento de teorias. ${ }^{15}$ Nesse sentido, a análise conceitual é ponto central para o avanço do conhecimento disciplinar em enfermagem referente aos elementos da prática, a exemplo os diagnósticos de enfermagem. ${ }^{11}$

O processo de análise de conceitos tem se baseado principalmente na abordagem proposta por Lorraine $\mathrm{O}$. Walker e Kay C. Avant, originalmente publicado em 1983, no qual as autoras adaptam o método de Wilson trazido da sociologia. ${ }^{16}$ Em 1989 , Beth L. Rodgers propôs uma abordagem para análise de conceito que valoriza o dinamismo e as interrelações, destacando que a análise do conceito não é imutável, mas dinâmica, variando com o contexto e com o tempo, o que sustenta claramente a ideia de desenvolvimento do conceito. ${ }^{14} \mathrm{O}$ desenvolvimento ocorre em ciclo evolutivo com três aspectos relevantes: significância, uso e aplicação do conceito. A significância refere-se a quando o conceito adquire um significado por servir a um relevante propósito humano em casos práticos atuais. $\mathrm{O}$ uso refere-se 
à definição e suas bases contextuais, como os atributos do conceito. Por sua vez, a aplicação do conceito concerne às características de conceitos em vários contextos, ao longo do tempo e em contextos específicos. ${ }^{14,17}$

A aplicação conceitual a um contexto específico pode ter relação com o constructo diagnóstico de enfermagem. Os diagnósticos da NANDA International Inc. são conceitos construídos em um sistema multiaxial, sendo também constituídos por focos diagnósticos. ${ }^{9}$ Nesta perspectiva, um conceito abrangente, como por exemplo o conforto, adquire relevância prática clínica por ser um foco da atenção da enfermeira estando referenciado ao fenômeno vivenciado pelo indivíduo, grupo ou comunidade. O diagnóstico de enfermagem como um conceito-chave é capaz de fornecer elementos para caracterização adequada do fenômeno e prover bases para resolução de problemas, permitir estimativas de vulnerabilidades e encaminhar a caracterização da promoção da saúde, o que torna a conjugação análise conceitual e pesquisa diagnóstica de enfermagem um promissor campo de desenvolvimento do conhecimento.

Os atributos e a expressão conceitual são essenciais para a definição do conceito, sendo utilizados para a construção da definição do diagnóstico de enfermagem. A partir da aplicação do conceito sua amplitude ou escopo tornam-se claros, revelando não somente as fortalezas do conceito, mas, também, suas limitações. ${ }^{14}$ No desenvolvimento do conceito os estudos de validação diagnóstica de enfermagem permitem o contínuo refinamento do núcleo conceitual do diagnóstico no interesse da prática clínica.

\section{MÉTODO}

Estudo de análise conceitual baseada no modelo evolucionário de Rodgers que, considerando o método empregado, se desenvolveu em sete etapas: identificação do conceito de interesse e expressões associadas; seleção da configuração e da amostra; coleta e gerenciamento dos dados; análise dos dados referentes às características do conceito; identificação de um exemplo do conceito; interpretação de resultados e; identificação de implicações para demais desenvolvimentos do conceito. ${ }^{14} \mathrm{~A}$ meta da análise de conceito é a produção de conhecimento necessário para a condução da fase 1 de validação de diagnósticos de enfermagem. ${ }^{11}$

A primeira etapa de identificação do conceito de interesse e expressões associadas, levou em conta a relevância do conceito nos estudos de natureza teórico-conceitual e as investigações classificatórias de representações diagnósticas associadas as taxonomias da Enfermagem.

A segunda etapa de seleção da configuração e amostra apropriada para a coleta de dados, incorporou as decisões ligadas ao método e que orientam a revisão da literatura, sendo elas: em relação ao período de tempo a ser examinado, as disciplinas produtoras das fontes e aos tipos de materiais a serem usados. ${ }^{14}$ Foram selecionados: artigos que atendessem aos elementos significativos da pergunta de pesquisa, publicados em inglês, espanhol ou português, sem um marco temporal inicial e o limite compreendendo o período da busca. A configuração disciplinar incorporou a Enfermagem em função da meta orientada para o diagnóstico de enfermagem. Quanto ao tipo foram captados artigos conceituais, teóricos e clínicos de desenhos qualitativos e quantitativos e revisões da literatura. Foram excluídas as cartas a editores.

Os títulos e resumos dos artigos recuperados na busca foram lidos e analisados por um dos revisores para identificar os potencialmente elegíveis para o estudo. Nas situações de dúvida os artigos permaneceram para a fase seguinte, que envolveu a leitura na íntegra de cada um dos artigos selecionados por dois revisores independentes para: a) confirmar a pertinência à pergunta de revisão e, em caso positivo, b) extrair os dados de interesse, o que são procedimentos específicos da próxima etapa.

A etapa de coleta e gerenciamento dos dados, a terceira do método de análise, incorporou os procedimentos de decomposição conceitual para extração dos elementos que compunham o conceito de conforto, sendo eles: atributos do conceito; base contextual (antecedentes e consequentes) do conceito e termos correlatos. Foi utilizado o organizador bibliográfico Endnote ${ }^{\circledR}$ com as informações das fontes analisadas e com os elementos do conceito de conforto. A busca bibliográfica foi realizada nos meses de novembro e dezembro de 2018. Para a organização dos achados foi construída uma planilha Office Exce ${ }^{\circledR}$ contendo o país no qual esse foi realizado; o ano em que foi publicado; o periódico de publicação; o método utilizado; o tema central, os principais achados do estudo e os mencionados elementos conceituais.

Na revisão integrativa a questão de busca foi inicialmente voltada para o campo da enfermagem cardiológica, especificamente para pessoas com insuficiência cardíaca, sendo ela: "Quais elementos do conceito de conforto são incorporáveis ao diagnóstico de enfermagem "Disposição para conforto melhorado" (00183) em pacientes com insuficiência cardíaca?". Esta foi elaborada a partir de adaptação do acrônimo PCC (População, Conceito e Contexto). ${ }^{18}$ Portanto, foram incorporados: População com insuficiência cardíaca, o Conceito de conforto e o Contexto de todas as áreas de cuidado em que fossem aplicados ao diagnóstico de enfermagem.

Após a determinação destes elementos os termos padronizados e seus sinônimos em português, espanhol e inglês foram buscados como descritores Medical Subject Headings (MESH), CINAHL Headings e Descritores em Ciências da Saúde, de acordo com a base de dados, utilizando o operador booleano "OR", como demonstrado na Tabela 1.

A busca bibliográfica foi conduzida no portal Regional da Biblioteca Virtual de Saúde (BVS), abrangendo as bases de dados científicas Literatura Latino-Americana e do Caribe em Ciências da Saúde (LILACS), Índice Bibliográfico Espanhol em Ciências da Saúde (IBECS), somado a busca no portal Pubmed da National Library of Medicine (NLM) que inclui a base de dados Medical Literature Analysis and Retrieval System Online (Medline), no portal de Periódicos da Capes, e ainda foram empregadas as bases de dados multidisciplinares: Web of Science, Index to 
Tabela 1. Logic Grid das estratégias de busca dos estudos nas bases de dados selecionadas.

\begin{tabular}{|c|c|c|}
\hline POPULAÇÃO & CONCEITO & CONTEXTO \\
\hline 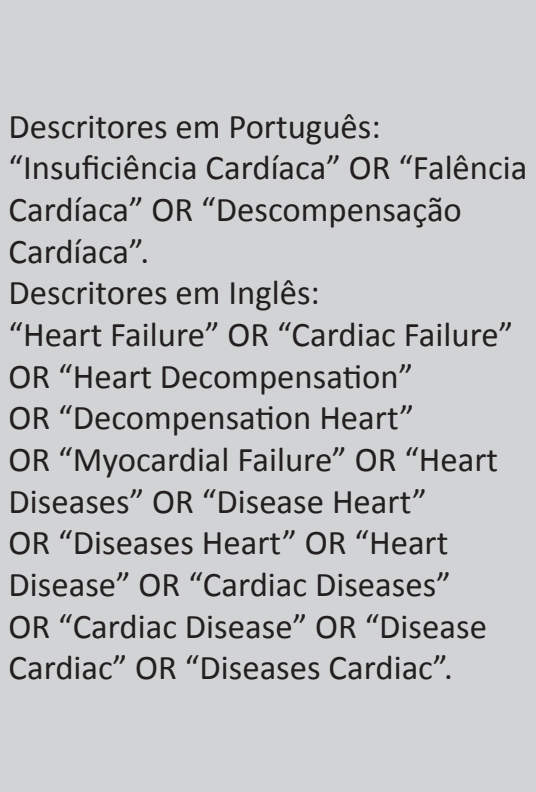 & 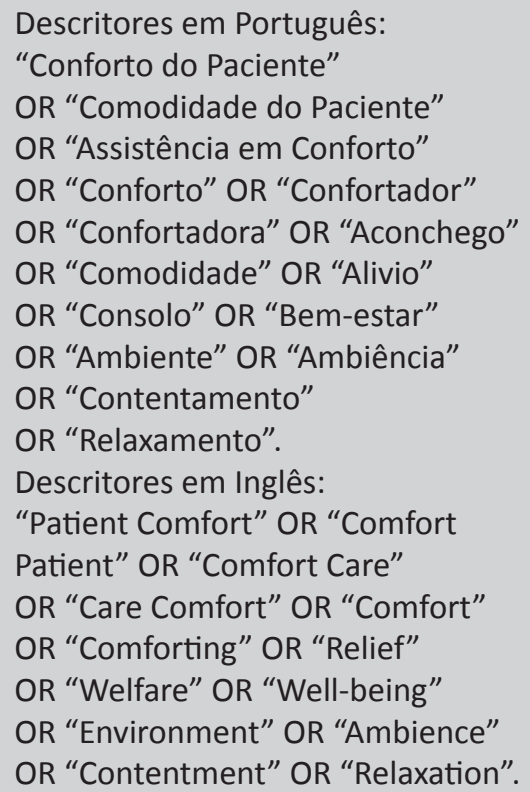 & $\begin{array}{l}\text { Descritores em Português: } \\
\text { "Diagnóstico de Enfermagem" } \\
\text { OR “Diagnósticos de Enfermagem". } \\
\text { Descritores em Inglês: } \\
\text { "Nursing Diagnosis" OR "Diagnosis } \\
\text { Nursing" OR "Diagnoses Nursing" } \\
\text { OR "Nursing Diagnoses". }\end{array}$ \\
\hline
\end{tabular}

Nursing and Allied Health Literature (CINAHL) - via EBSCOhost e Scopus (Elsevier). Utilizou-se ainda o Portal de Revistas Eletrônicas Scientific Electronic Library Online (Scielo).

Em pré-análise dos resultados da busca, verificou-se que a inclusão da População com insuficiência cardíaca (acrônimo P) limitaria sobremaneira o material disponível para análise de conceito. Diante disso, os pesquisadores optaram por incorporar os demais artigos que retornaram e pudessem atender aos critérios de estudos elegíveis (limites temporais, tipo de artigo, configuração disciplinar e idioma de publicação); então, assumindo a inclusão do artigo independente da condição de conforto abordada ter ou não relação com a insuficiência cardíaca.

Os resultados dos estudos retornados da revisão de literatura estão dispostos na Figura 1.

A quarta etapa de análise dos dados referentes às características do conceito, foi desenvolvida usando evidências extraídas da revisão integrativa, estando sustentada na perspectiva evolutiva do conceito e ancorada em considerações estruturais taxonômicas do diagnóstico de enfermagem. Amparada no método de análise evolutiva, foi dada atenção especial aos seguintes indicadores: conceito, evolução temporal, atributos, antecedentes, consequentes e termos substitutos, correlacionando-os com o diagnóstico de enfermagem.

A quinta etapa de identificação de um exemplo do conceito foi desenvolvida na construção de um caso expressivo da "Disposição para conforto melhorado" (00183) em pessoa com Insuficiência Cardíaca. Foram incorporados: resultados da análise de conceito, o conteúdo atual do diagnóstico de enfermagem ${ }^{9}$ e os padrões típicos do diagnóstico vivenciados na experiência clínica dos pesquisadores. $\mathrm{O}$ caso foi redigido em estrutura comum em documentação clínica de dados históricos e de avaliação no Brasil, contendo: dados de identificação, dados pregressos da doença e de tratamento médico e estado atual com anotação no formato SOAP (\#Subjetivo, \#Objetivo, \#Avaliação e \#Plano). ${ }^{19,20}$

A etapa seis de interpretação de resultados e a última de identificação de implicações para demais desenvolvimentos do conceito foram conduzidas na interpretação dos achados e extrapolação para o contexto do diagnóstico de enfermagem de promoção à saúde. Em termos redacionais foram incorporadas especialmente na discussão.

\section{ANÁLISE DO CONCEITO DE CONFORTO}

A base das evidências para análise do conceito utiliza os resultados dos estudos retornados da revisão da literatura.

\section{Evolução temporal e definição do Conceito}

A primeira etapa da análise relaciona-se a escolha do conceito, e como já elucidado, o conceito conforto é importante e diretamente ligado à prática de enfermagem. A segunda etapa metodológica da análise conceitual que trata da seleção da configuração e amostra apropriada para a coleta de dados e dos documentos indicou a incorporação de dezenove estudos nas tipologias: estudos de análise de conceito, pesquisas teóricas e estudos de sustentação empírica.

A terceira etapa da análise referente a coleta e gerenciamento dos dados explorou os elementos extraídos do processo analítico do conceito que são desenvolvidos na perspectiva histórico-desenvolvimental do conforto. Do ponto de vista histórico, observa-se que o conforto enquanto conceito tem sua evolução sofrendo interferência do tempo histórico vivenciado na 


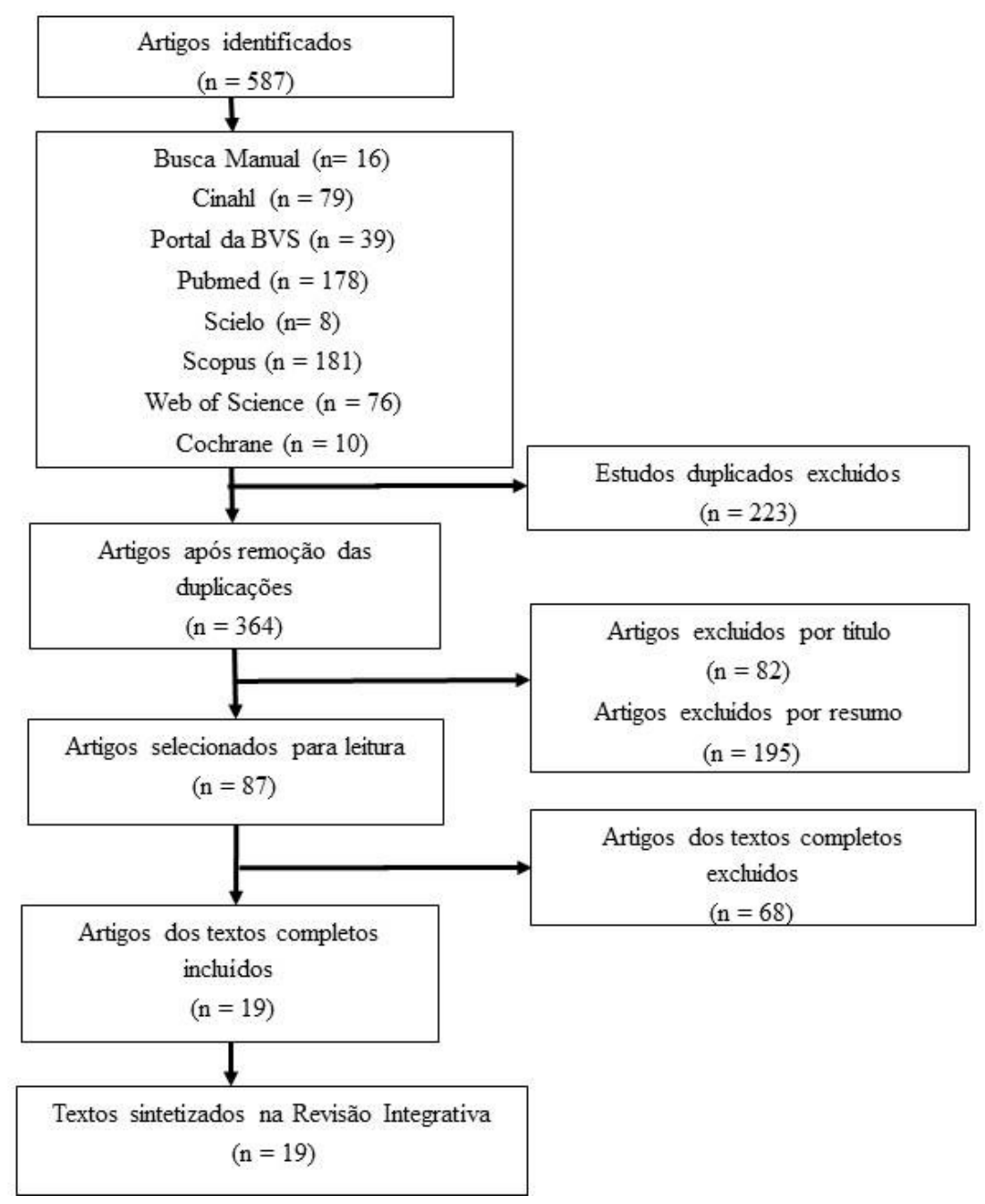

Figura 1. Fluxograma de estudos identificados e selecionados para inclusão na revisão

sociedade à época. Perpassou desde um objetivo a ser atingido pela enfermagem até um resultado para o paciente. Florence Nightingale, já abordava o conforto como meta do cuidado. ${ }^{21}$

No período de 1900 a 1929, o conforto era considerado a meta central e um imperativo moral da enfermagem, sendo ofertado aos pacientes geralmente em domicílio por enfermeiras. As ações de conforto eram essencialmente físicas que visava manter o paciente bem posicionado e confortável. Também era de natureza mental, alcançado a partir do fornecimento de conforto físico e modificação do ambiente..$^{22}$

Entre 1930 a 1959, período marcado por depressão econômica, a falta de profissionais durante a guerra e o consequente desemprego, deslocaram enfermeiras dos domicílios para o sistema hospitalar. Essa institucionalização das práticas de saúde causou aumento do controle médico, subordinando o conforto do paciente às metas médicas. Consequentemente, o conforto deixa de ser a meta primordial da enfermagem. ${ }^{22}$

De 1960 a 1980, devido à expansão tecnológica, foi reduzido o tempo de contato direto da enfermeira com os pacientes, sendo o trabalho da enfermeira deslocado para monitorar equipamentos e desenvolver atividades técnicas e de gerenciamento, passando as medidas de conforto a serem executadas por outros membros da equipe de enfermagem. Entretanto, o emprego de habilidades interpessoais e de comunicação passam a incorporar o processo de conforto, inclusive com a participação do paciente na garantia do seu próprio conforto. ${ }^{22,23}$

A partir dos anos de 1990, estudos de análise de conceito e a consequente teorização permitiram a inserção no campo do conhecimento disciplinar e produção de linguagem própria. . $^{3,4,7}$ Assim, em 2006, foi incluído na Taxonomia II da NANDA-I o diagnóstico de "Disposição para conforto melhorado" (00183) e em 2008 o "Conforto prejudicado" (00214). ${ }^{9}$

Com a institucionalização dos cuidados em um contexto hospitalocêntrico e numa perspectiva biologicista, provavelmente, o foco da avaliação da enfermagem intensifica-se para fenômenos indesejáveis que representam problemas, como por exemplo, os diagnósticos de enfermagem do domínio do conforto: dor aguda (00132), a "náusea" (00134), a "dor crônica" (00133) e o "conforto 
prejudicado" (00214). Há que se considerar ainda, a perspectiva social do conceito de conforto trazida na literatura. Consumidores do conforto, de classes sociais diferentes, são submetidos a distintas condições de atendimento de suas necessidades de conforto. Especialmente em países mais pobres, a classe social com menor poder aquisitivo e mais limitado acesso aos serviços de saúde de excelência gozam de um nível de conforto limitado. ${ }^{23}$

A partir do entendimento das mudanças históricas e decomposição do conteúdo foram localizados núcleos conceituais de definições. De forma genérica, as definições de conforto foram distribuídas em três categorias de núcleo conceitual: ligadas ao problema/doença; ligadas ao bem-estar/promoção da saúde e ligadas a metas da profissão, que são sintetizadas no Quadro 1.

As definições que incorporaram elementos ligados ao problema/doença apontaram que o desconforto, condição antagônica ao conforto, deve ser aliviado, sendo esse um dos objetivos da enfermagem, a partir da aplicação de cuidados em resposta às necessidades apresentadas pela pessoa, e com a adoção de estratégias alinhadas às competências profissionais da enfermagem. Esta perspectiva de estado de agravo ou desarmonia representa uma contribuição para a definição de conforto em oposição ao que de fato seria o conforto.

As definições que incorporaram núcleos ligados ao bem-estar/promoção da saúde delimitaram de forma conceitual o que de fato representaria o conforto. Termos como harmonia, transcendência, bem-estar, fortalecimento, encorajamento, dentre outros, expressam uma dimensão positiva do conforto estando subordinados à posição epistemológica dos autores dos estudos que produziram as definições. De modo sintético, o conforto pode ser entendido como estado em que estão satisfeitas as necessidades básicas relativas aos estados de alívio, tranquilidade e transcendência.

Apesar da tentativa universalista de definir o conforto, não se deve perder de vista que a ideia do nível de conforto pode variar em função da cultura, do sexo, das circunstâncias que desencadeiam a necessidade de conforto, estado de saúde, da experiência da doença, do ambiente, entre outros. ${ }^{24}$

\section{Atributos do Conceito Conforto}

A identificação dos atributos de um conceito representa a principal realização de uma análise conceitual, em outras palavras, são pistas para as características definidoras e definições reais do conceito de interesse. ${ }^{14}$ No Quadro 2 são expostos os atributos do conceito inferidos nos estudos analisados no presente estudo.

Os estudos convergem para atributos de satisfação, bem-estar e melhora na qualidade de vida. $O$ bem-estar se materializa em sensações de natureza física, psicológica, espiritual e ambientais, o que recorta as dimensões mais objetivas do conforto enquanto um fenômeno.

Em termos de estado, o conforto pode ser pontuado como alívio, tranquilidade e transcendência, desenvolvendo-se em quatro contextos: o contexto físico, que refere-se às sensações corporais; o contexto sociocultural às relações interpessoais, familiares e sociais; o contexto psicoespiritual à consciência de si, incluindo a autoestima, o auto-conceito, sexualidade e sentido de vida; e o contexto ambiental, que envolve aspectos como a luz, barulho, equipamento e elementos naturais ou artificiais do meio. . $^{3,4,23,24}$

Quadro 1. Síntese das definições extraídas da literatura acerca do fenômeno conforto.

\section{Definições}

Núcleos conceituais ligados ao problema/doença: Cuidado para tornar o outro livre de dores e desconforto. ${ }^{10,24,25,26,27,28}$ Alívio do desconforto. ${ }^{1,3,4,24,25,26,28,29,30}$ Estado isento de dor. ${ }^{31}$ Alívio da dor, angústia mental ou outro desconforto. . $^{3,4,29}$

Núcleos conceituais ligados ao bem-estar/promoção da saúde: Estado de ter atendido as necessidades humanas básicas de

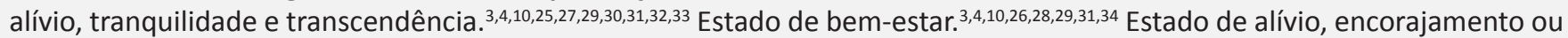
consolo. ${ }^{25,30,31}$ Estado de harmonia, resultado da integração corpo-mente-espírito. ${ }^{3,24,25,31}$ Bem-estar mental e físico. ${ }^{3,25,30,31,34}$ Estado de conforto físico. ${ }^{22,23}$ Estado de conforto mental. ${ }^{22,23}$ Fortalecimento, encorajamento, apoio. ${ }^{10,25,26,29}$ Estado de tranquilidade ${ }^{3,4,29,30}$ Núcleos conceituais ligados a metas da profissão: Estado final das ações terapêuticas de enfermagem para um paciente ${ }^{22,23,24,28}$

Quadro 2. Síntese dos atributos extraídos da literatura acerca do fenômeno conforto.

\section{Atributos}

Transcendência (superação de problemas ou dor). 1,3,4,10,24,25,29,30,31,32,33,35 Sensação de tranquilidade ou satisfação. ${ }^{3,24,30,31,33}$ Capacidade em ter e fazer suas próprias atividades..$^{1,24,31,32}$ Sensação de integralidade, funcionalidade e normalidade. ${ }^{10,24,31}$ Desfrutar de interações pessoais e sensação de bem-estar físico, psicológico e espiritual. ${ }^{1,24,25,31,33,35}$ Conforto que incorpora necessidades físicas, psicoespirituais, sociais e ambientais. ${ }^{3,4,27,29,30}$ Possuir recursos materiais ou financeiros. ${ }^{24}$ Aspecto central para satisfazer as necessidades humanas. ${ }^{1,26,27}$ Cuidado holístico. ${ }^{3,4,26,27,29,30,33}$ Qualidade de vida. ${ }^{10,33}$ Sensação de bem-estar mental e físico. ${ }^{3,25,27,28,31}$ Estar bem consigo mesmo e com outras pessoas. ${ }^{1,10,31}$ Satisfação das necessidades humanas básicas. ${ }^{3,25,27,29,30,32,33,34}$ Sentir-se bem. ${ }^{34}$ Alívio, tranquilidade e calma. ${ }^{25,28,32,34,35}$ Estado de facilidade ou contentamento. ${ }^{30}$ Esperança e confiança. ${ }^{26,28}$ 
Quadro 3. Síntese dos antecedentes, consequentes e termos substitutos extraídos da literatura acerca do conforto.

\section{Antecedentes}

Efeitos indesejáveis. ${ }^{3,4,10,24,26}$ Desconforto. ${ }^{1,3,4,10,24,25,26,28,29,30,31,32,33,35}$ Necessidades fisiológicas insatisfeitas. . $^{3,4,27,29,30,31}$ Dor. ${ }^{1,24,26,36}$ Desconforto psicológico, físico e espiritual. ${ }^{1,3,24,25,31}$ Estresse. ${ }^{36}$ Ansiedade. ${ }^{36}$ Necessidade de conforto não satisfeitas (alívio, facilidade e transcendência). ${ }^{3,4,30}$ Angustia. ${ }^{33}$ Sofrimento. ${ }^{33}$

\section{Consequentes}

Necessidades humanas básicas satisfeitas (alívio, facilidade e transcendência). 1,3,4,10,27,29,30,31,32,33,36 Desconforto específico aliviado..$^{3,4,24,25,29,30,31,33,35}$ Estar sem dor. ${ }^{24,31}$ Sentir-se bem consigo mesmo. ${ }^{3,24,33}$ Conforto psicológico, físico e social. ${ }^{1,3,10,23,25}$ Manutenção de atividades. ${ }^{1,4,24,29,32}$ Fazer o que deseja. ${ }^{24,31}$ Sentir satisfação com o tratamento. ${ }^{24,27,33}$ Alívio da dor. ${ }^{1,24,28,34,36}$ Ausência de efeitos indesejáveis. ${ }^{3,31,34}$ Alívio da ansiedade. ${ }^{33,36}$ Alívio do estresse. ${ }^{36}$ Aumento do nível de conforto. ${ }^{3,4,26,27,29,36}$ Ausência de condições de desconforto. ${ }^{4,10,26,29}$ Sentir-se fortalecido, revigorado. ${ }^{30}$ Melhora na qualidade de vida. ${ }^{10,33}$ Melhor interação paciente-profissional da saúde. ${ }^{26,27,33}$

\section{Termos Substitutos}

Bem-estar. ${ }^{1,4,24,26,28,29,31,32,34}$ Tranquilidade. ${ }^{32,34}$ Alívio do desconforto. ${ }^{3,4,10,24,26,27,28,29,30,36}$ Calma. ${ }^{24,31,34}$ Autoestima. ${ }^{24}$ Ausência de perturbações. ${ }^{24}$ Experiência agradável. ${ }^{3,10}$ Alívio da dor. ${ }^{10,26,33}$ Sensação de paz interior. ${ }^{10}$ Medidas de apoio. ${ }^{33}$

\section{Antecedentes, Consequentes e termos substitutos do Conforto}

O Quadro 3 representa a síntese dos antecedentes, consequentes e termos substitutos do fenômeno conforto mencionados nos estudos analisados.

Os antecedentes compreendem os acontecimentos que precedem o conceito de interesse e, a partir deles, faz-se possível identificar os diferentes contextos em que uma definição pode estar sendo utilizada, em contrapartida, os consequentes direcionam os resultados para os acontecimentos que surgiram após a aplicação do conceito em análise. ${ }^{37}$ Os antecedentes sintetizados na presente pesquisa foram aqueles que poderiam estar relacionados a uma perspectiva de disposição para melhora do conforto, sendo por isso de natureza negativa.

Os consequentes carregam em si a dimensão de resultados ou meta a ser alcançada com a resolução dos problemas ligados ao não atendimento ou desequilíbrio do conforto ou ainda ao fim atingido pela pessoa que apresenta uma "Disposição para conforto melhorado" (00183).

Quanto aos termos substitutos, estão relacionados a palavras que sugerem o alívio dos sintomas manifestos ou a um aspecto do conforto, como os de satisfação do paciente expresso por alívio do desconforto e bem-estar, este, diretamente ligado ao termo conforto observado na análise.

A caracterização de um termo como antecedente ou consequente foi baseada na decisão consensual dos autores que levaram em consideração as evidências dos estudos analisados. Entretanto, as decisões de natureza conceitual estão sempre condicionadas a aspectos de semântica e conceitualização dos analistas, o que confere uma incontornável subjetividade de interpretação.

\section{Caso exemplo da "Disposição para conforto melhorado" (00183)}

Sr. J.M.R., 60 anos, sexo masculino. \#S: Comorbidades relatadas: hipertensão arterial e Insuficiência Cardíaca. Paciente relata falta de ar, dificuldade em realizar as atividades de vida diária, edema em membros inferiores e cansaço aos médios esforços, devido a insuficiência. Expressa desejo em melhorar sua qualidade de vida, explicitado em sua fala: "sinto desejo de melhorar o cansaço e essa falta de ar que sinto, mesmo que para isso tenha de praticar alguma atividade e me tornar mais ativo. Gostaria de satisfazer minhas necessidades do dia-a-dia, sem precisar de ajuda. Acho que se voltar a frequentar a igreja, irei me sentir bem comigo e com as outras pessoas que eram próximas, pois quero muito isso para mim. Acho que no fundo quero ajudar de algum modo a me sentir mais aliviado e tranquilo com tudo isso que estou vivendo". \#O: Nível de consciência alerta, orientado no tempo e espaço, responsivo às solicitações verbais. Hipocorado (+2/+4), hidratado, acianótico e anictérico. Algo dispneico, ventilando com suporte de cateter nasal com $\mathrm{O} 2$ á $2 \mathrm{~L} / \mathrm{min}$ com ausculta pulmonar normal. A ausculta cardíaca com bulhas normofonéticas em dois tempos. Hemodinâmica: normotenso, tendendo a bradicardia. SatO2=95\%. Afebril. Dieta por via oral com moderada aceitação. Abdome globoso, peristáltico, indolor a palpação. Membros superiores livres de edema. Membros inferiores edemaciados (+2/+4), ambos perfusos e aquecidos. Funções vesico-intestinais presentes espontaneamente. \#A: DE: Disposição para conforto melhorado, caracterizado por expressar desejo de aumentar o conforto, melhorar a resolução das queixas, motivação e desejo em melhorar a satisfação das necessidades humanas básicas, melhorar a sensação de bem-estar espiritual, motivação e condição de transcender ao problema ou dor, motivação, desejo e condição para realizar suas próprias atividades. \#P: Foi motivado a permanecer buscando atender suas necessidades referentes ao conforto na dimensão física, encorajando-o ao autocuidado e planejando metas para a realização de suas atividades de vida diária.

\section{Incorporação dos elementos da análise conceitual na estrutura do diagnóstico de enfermagem "Disposição para conforto melhorado" (00183)}

A análise de conceito forneceu elementos de evidência para uma proposta de modificações na estrutura do diagnóstico "Disposição para conforto melhorado" (00183) da Taxonomia da 
Tabela 2. Síntese dos elementos derivados da análise de conceito do diagnóstico de enfermagem "Disposição para conforto melhorado" (00183).

\begin{tabular}{|c|c|}
\hline Domínio & Domínio 12 - Conforto \\
\hline Título & Disposição para conforto melhorado \\
\hline Definição & $\begin{array}{l}\text { Motivação, desejo, ou condição de melhora do conforto em suas dimensões física, } \\
\text { psicoespiritual, ambiental e/ou social. }\end{array}$ \\
\hline Características Definidoras & $\begin{array}{l}\text { Expressa desejo de aumentar a sensação de contentamento; Expressa desejo de } \\
\text { aumentar o conforto; Expressa desejo de aumentar o relaxamento; Expressa desejo } \\
\text { de melhorar a resolução das queixas; Expressa motivação, desejo ou condição para } \\
\text { melhora na satisfação das necessidades humanas; Expressa motivação, desejo ou } \\
\text { condição para melhorar a sensação de bem-estar físico, psicológico e espiritual; } \\
\text { Expressa motivação, desejo ou condição para alcançar a transcendência ao problema } \\
\text { ou dor; Expressa motivação, desejo ou condição para realizar suas próprias atividades. }\end{array}$ \\
\hline $\begin{array}{l}\text { Este diagnóstico está classificado } \\
\text { na Classe } 1 \text { (Conforto físico), na } \\
\text { Classe } 2 \text { (Conforto ambiental) e na } \\
\text { Classe } 3 \text { (Conforto social). }\end{array}$ & \\
\hline
\end{tabular}

NANDA-I (2018), propondo a substituição da atual definição de "Padrão de conforto, alívio e transcendência nas dimensões física, psicoespiritual, ambiental e/ou social que pode ser melhorado" por "Motivação, desejo ou condição de melhora do conforto em suas dimensões física, psicoespiritual, ambiental e/ou social, como a incorporação de algumas características definidoras, que é apresentada na Tabela 2.

\section{CONCLUSÕES E IMPLICAÇÕES PARA A PRÁTICA}

A análise do conceito de conforto no método evolucionário demonstrou que mudanças conceituais e na estrutura diagnóstica permitiram atualizar o diagnóstico de "Disposição para conforto melhorado"(00183). Os elementos verificados na literatura abordam uma perspectiva orientada tanto para o bem-estar quanto para as alterações caracterizadas no desconforto, o que permitiu a extração de definições, atributos, antecedentes, consequentes e termos correlatos que contribuíram para clarificação do diagnóstico de enfermagem. Considerando a evolução temporal do conforto no âmbito da profissão de enfermagem, a abordagem evolucionária de análise do conceito mostrou-se ser apropriada.

O desenvolvimento e evolução das análises diagnósticas em enfermagem é aspecto central para a disciplina e necessária para o avanço do nível de evidência (LOE) dos diagnósticos de enfermagem. Nesse sentido, os autores entendem que na perspectiva evolucionária, os resultados desta pesquisa já fornecem elementos para a realização de estudos de validação diagnóstica por experts e validação clínica da "Disposição para conforto melhorado" (00183).

\section{FINANCIAMENTO}

Bolsa de mestrado concedida à Fundação de Amparo a Pesquisa do Estado do Rio de Janeiro. Processo nํ200.839/2019 (242.621), a partir de abril de 2019.

\section{CONTRIBUIÇÕES DOS AUTORES}

Desenho do estudo. Aquisição, análise de dados e interpretação dos resultados. Redação e revisão crítica do manuscrito. Aprovação da versão final do artigo. Responsabilidade por todos os aspectos do conteúdo e a integridade do artigo publicado. Cristiane Soares Carius Nogueira Pereira, Marcos Antônio Gomes Brandão. Interpretação dos resultados e revisão crítica do manuscrito. Aprovação da versão final do artigo. Responsabilidade por todos os aspectos do conteúdo e a integridade do artigo publicado. Claudia Angélica Mainenti Ferreira Mercês, Rafael Oliveira Pitta Lopes, Jackeline Felix de Souza, Jaqueline da Silva Soares Souto.

\section{EDITOR ASSOCIADO}

Eliane Tatsch Neves

\section{REFERÊNCIAS}

1. Apóstolo JLA. O conforto nas teorias de enfermagem: análise do conceito e significados teóricos. Rev Referência. 2009;(9):61-7.

2. Pinto SMO, Berenguer SMAC, Martins JCA. Is impaired comfort a nursing diagnosis? Int J Nurs Knowl. 2016 out;27(4):205-9. http://dx.doi. org/10.1111/2047-3095.12121. PMid:26459147.

3. Kolcaba KY. Holistic comfort: operationalizing the construct as a nursesensitive outcome. ANS Adv Nurs Sci. 1992 set;15(1):1-10. http://dx.doi. org/10.1097/00012272-199209000-00003. PMid:1519906.

4. Kolcaba KY. A theory of holistic comfort for nursing. J Adv Nurs. 1994 jun;19(6):1178-84. http://dx.doi.org/10.1111/j.1365-2648.1994.tb01202.x. PMid:7930099.

5. Kolcaba K, Steiner R. Empirical evidence for the nature of holistic comfort. J Holist Nurs. 2000 mar;18(1):46-62. http://dx.doi. org/10.1177/089801010001800106. PMid:11847691.

6. Morse JM. On comfort and comforting. Am J Nurs. 2000;100(9):34-7. PMid:11002789.

7. Morse JM, Bottorff JL, Hutchinson S. The phenomenology of comfort J Adv Nurs. 1994Jul;20(1):189-95. http://dx.doi.org/10.1046/j.13652648.1994.20010189.x. PMid:7930120. 
8. Garcia TR, organizador. Classificação Internacional da Prática de enfermagem (CIPE): versão 2017. Porto Alegre: Artmed; 2018. 254 p.

9. Herdman H, Kamitsuru S. Diagnósticos de enfermagem da NANDA-I definições e classificação - 2018/2020. 11. ed. Porto Alegre: Art Med; 2018.

10. Siefert ML. Concept analysis of comfort. Nurs Forum. 2002out;37(4):16-23. http://dx.doi.org/10.1111/j.1744-6198.2002.tb01288.x. PMid:12592834

11. Lopes MVO, Silva VM. Métodos avançados de validação de diagnósticos de enfermagem. In: Herdman TH, organizador. PRONANDA: programa de atualização em diagnósticos de enfermagem: conceitos básicos. Porto Alegre: Artmed/Panamericana; 2016. p. 87-132.

12. Duncan C, Cloutier JD, Bailey PH. Concept analysis: the importance of differentiating the ontological focus. J Adv Nurs. $2007 \mathrm{abr} ; 58$ (3):293-300. http://dx.doi.org/10.1111/j.1365-2648.2007.04277.x. PMid:17474918.

13. Dau S. Ciência: pesquisa, métodos e normas. 2. ed. Juiz de Fora: Alexandria; 2006.

14. Rodgers BL. Concept analysis: an evolutionary view. In: Rodgers BL, Knafl KA, organizadores. Concept development in nursing: foundations, techniques, and applications. 2nd ed. Philadelphia: W. B. Saunders 2000. p. 77-102.

15. Bousso RS, Poles K, Cruz DALM. Nursing concepts and theories. Rev Esc Enferm USP. 2014 fev;48(1):141-5. http://dx.doi.org/10.1590/ S0080-623420140000100018. PMid:24676120.

16. Walker LO, Avant KC. Strategies for theory construction in nursing. 6th ed. New York: Pearson; 2018. 262 p.

17. Weaver K, Mitcham C. Nursing concept analysis in North America: state of the art. Nurs Philos. 2008jul;9(3):180-94. http://dx.doi.org/10.1111/ j.1466-769X.2008.00359.x. PMid:18582294.

18. The Joanna Briggs Institute. Joanna Briggs Institute reviewers' manual: 2015 edition [Internet]. South Australia: The Joanna Briggs Institute; 2015 [citado 2017 jul 24]. Disponível em: http://joannabriggs.org/ assets/docs/sumari/Reviewers-Manual_Methodology-for-JBI-ScopingReviews_2015_v1.pdf

19. Jaroudi S, Payne JD. Remembering Lawrence Weed: a pioneer of the SOAP note. Acad Med. 2019 jan;94(1):11. http://dx.doi.org/10.1097/ ACM.0000000000002483. PMid:30585813.

20. Ministério da Saúde (BR). Sistema E-SUS: atenção básica. Brasília: Ministério da Saúde; 2019

21. Wurzbach ME. Comfort and nurses' moral choices. J Adv Nurs. 1996 ago;24(2):260-4. http://dx.doi.org/10.1046/j.1365-2648.1996.01985.x. PMid:8858428.

22. Mcllveen $\mathrm{KH}$, Morse $\mathrm{J}$. The role of comfort in nursing care: 1900-1980. Clin Nurs Res. 1995 maio;4(2):127-48. http://dx.doi. org/10.1177/105477389500400202. PMid:7757022.

23. Mussi FC. Conforto e lógica hospitalar: análise a partir da evolução histórica do conceito conforto na enfermagem. Acta Paul Enferm. 2005 mar;18(1):72-81. http://dx.doi.org/10.1590/S0103-21002005000100010.
24. Mussi FC, Friedlander MR, Arruda EN. Os significados da palavra conforto segundo a perspectiva do paciente com infarto agudo do miocárdio. Rev Lat Am Enfermagem. 1996 dez;4(3):19-39. http://dx.doi. org/10.1590/S0104-11691996000300003.

25. Malinowski A, Stamler LL. Comfort: exploration of the concept in nursing J Adv Nurs. 2002;39(6):599-606. http://dx.doi.org/10.1046/j.13652648.2002.02329.x. PMid:12207758.

26. Morse JM. Comfort: the refocusing of nursing care. Clin Nurs Res. 1992 fev;1(1):91-106. http://dx.doi.org/10.1177/105477389200100110. PMid:1493486.

27. Kolcaba K. Evolution of the mid range theory of comfort for outcomes research. Nurs Outlook. 2001 mar;49(2):86-92. http://dx.doi.org/10.1067/ mno.2001.110268. PMid:11309563.

28. Tutton E, Seers K. An exploration of the concept of comfort. J Clin Nurs. 2003 ago;12(5):689-96. http://dx.doi.org/10.1046/j.13652702.2003.00775.x. PMid:12919215

29. Kolcaba KY, Kolcaba RJ. An analysis of the concept of comfort. J Adv Nurs. 1991 nov;16(11):1301-10. http://dx.doi.org/10.1111/j.1365-2648.1991. tb01558.x. PMid:1753026.

30. Kolcaba KY. A taxonomic structure for the concept comfort. J Nurs Scholarsh 1991 dez;23(4):237-40. http://dx.doi.org/10.1111/j.1547-5069.1991. tb00678.x. PMid:1937522.

31. Mussi FC. Conforto: revisão de literatura. Rev Esc Enferm USP. 1996 ago;30(2):254-66. http://dx.doi.org/10.1590/S0080-62341996000200006. PMid:8920409.

32. Silva FVF, Silva LF, Rabelo ACS. Processo de enfermagem no conforto do paciente com insuficiência cardíaca no domicílio. Aquichan. 2015 jun;15(1):116-28. http://dx.doi.org/10.5294/aqui.2015.15.1.11.

33. Pinto S, Caldeira S, Martins JC, Rodgers B. Evolutionary analysis of the concept of comfort. Holist Nurs Pract. 2017 jul/ago;31(4):243-52. http://dx.doi.org/10.1097/HNP.0000000000000217. PMid:28609409.

34. Silva CRL, Carvalho V, Figueiredo NMA. Predicações de conforto na perspectiva de clientes e de enfermeiros. Cogitare Enferm. 2011 jan mar;16(1):49-55. http://dx.doi.org/10.5380/ce.v16i1.21111.

35. Mendes RS, Cruz AM, Rodrigues DP, Figueiredo JV, Fialho AVM. Teoria do conforto como subsídio para o cuidado clínico de enfermagem. Cienc Cuid Saude. 2016 abr/jun;15(2):390-5. http://dx.doi.org/10.4025/ cienccuidsaude.v15i2.27767.

36. Puchi C, Paravic-klijn T, Salazar A. Comfort theory as a theoretical framework applied to a clinical case of hospital at home. Holist Nurs Pract. 2018 set/ out;32(5):228-39. http://dx.doi.org/10.1097/HNP.0000000000000275. PMid:30113956.

37. Monteiro MCM, Holanda VR, Melo GP. Análise do conceito parto humanizado de acordo com o método evolucionário de Rodgers. Rev Enferm Cent-Oeste Min [Internet]. 2017; [citado 2017 jul 24]; 7:e1885. Disponível em: http://seer.ufsj.edu.br/index.php/recom/article/view/1885 